Preprint of the paper

"High Order Shape Design Sensitivity: A Unified Approach"

F. Navarrina, S. López, I. Colominas, E. Bendito, M. Casteleiro (2000)

Computer Methods in Applied Mechanics \& Engrng., 188 , 681-696.

http:/caminos.udc.es/gmni 


\title{
High Order Shape Design Sensitivity: A Unified Approach
}

\author{
F. Navarrina ${ }^{\text {a }}$, S. López-Fontán ${ }^{\text {a }}$, I. Colominas ${ }^{a}$, E. Bendito ${ }^{\mathrm{b}}$, \\ M. Casteleiro ${ }^{\text {a }}$
}

a Universidad de La Coruña, Dep. de Métodos Matemáticos y de Representación, Escuela Técnica Superior de Ingenieros de Caminos, Canales y Puertos, Campus de Elviña, 15192 La Coruña, Spain

b Universidad Politécnica de Cataluña, Dep. de Matemática Aplicada III, Escuela Técnica Superior de Ingenieros de Caminos, Canales y Puertos, Campus Norte UPC, 08034 Barcelona, Spain

\begin{abstract}
Three basic analytical approaches have been proposed for the calculation of sensitivity derivatives in shape optimization problems. The first approach is based on differentiation of the discretized equations [1-3]. The second approach is based on variation of the continuum equations $[1,4,5]$ and on the concept of material derivative. The third approach [6] is based upon the existence of a transformation that links the material coordinate system with a fixed reference coordinate system. This is not restrictive, since such a transformation is inherent to FEM and BEM implementations.

In this paper we present a generalization of the latter approach on the basis of a generic unified procedure for integration in manifolds. Our aim is to obtain a single, unified, compact expression to compute arbitrarily high order directional derivatives, independently of the dimension of the material coordinates system and of the dimension of the elements. Special care has been taken on giving the final results in terms of easy-to-compute expressions, and special emphasis has been made in holding recurrence and simplicity of intermediate operations. The proposed scheme does not depend on any particular form of the state equations, and can be applied to both, direct and adjoint state formulations. Thus, its numerical implementation in standard engineering codes should be considered as a straightforward process. As an example, a second order sensitivity analysis is applied to the solution of a $3 \mathrm{D}$ shape design optimization problem.
\end{abstract}

Keywords: Shape Sensitivity, Sensitivity Analysis, Shape Optimization, Optimization, Integration in Manifolds, Finite Element Method 


\section{INTRODUCTION}

Most of analysis and design problems in engineering involve solving systems of partial differential equations (PDEs). Currently, the most powerfull and widely used methods for solving PDEs are the so-called integral methods, such as the Finite Element (FEM) and the Boundary Element (BEM) methods. The sensitivity analysis of this kind of methods requires taking derivatives of functions defined through integration. In fixed-geometry problems, the integration domains remain unchanged during the optimization process, and sensitivity analysis is usually performed by analytical techniques. However, integration domains are variable in shape optimization. This creates important additional difficulties [1] that have generally been overcome by employing finite difference approximations [7].

\section{STATEMENT OF THE PROBLEM}

According to the methology proposed by Navarrina and Casteleiro $[8,9]$ the first step in the statement of a design optimization problem is the definition of the criteria that will allow to decide whether a candidate design is acceptable or not, as much as to select the preferable design among the acceptable ones. The admissibility conditions are normally expressed by means of equality constraints $(h(\boldsymbol{\gamma})=0)$ and inequality constraints $(g(\boldsymbol{\gamma}) \leq 0)$ that must be verified by the final design. On the other hand, the quality of different acceptable designs can be normally compared by means of an adequate objective function $(f(\boldsymbol{\gamma}))$, defined in such a way that a lower value of the function is associated to a preferred design. The values $(\boldsymbol{\gamma})$, in terms of which the constraints and the objective function are defined, are called control variables of the problem.

The second step is the definition of a design parametric model, that for given values of the selected design constants $(\boldsymbol{c})$ and design variables $(\boldsymbol{x})$ will determine the corresponding value of the fundamental properties $(\boldsymbol{\varphi})$ that describe the nature of the object to be designed and its interactions with the environment. Hence, the design variables $(\boldsymbol{x})$ are the primal variables of the problem, that is, the unknown parameters which optimal value must be found.

It is obvious that some of the control variables $(\boldsymbol{\gamma})$ might be expressed directly in terms of the fundamental properties $(\boldsymbol{\varphi})$. However, in most of engineering design problems, an important part of the control variables will not depend directly on the properties of the design itself, but on the so-called state variables $(\boldsymbol{\omega})$ that describe the behaviour of the design in construction, service or fail conditions. Since the underlying physical phenomena must be analyzed, the third step is the definition of an analysis model, that for given values 
of the fundamental properties $(\varphi)$ will allow to compute the state variables $(\boldsymbol{\omega})$. Different analysis models could be proposed to analyze the same physical phenomena, and each one should require specific input data $(\boldsymbol{\alpha})$, that must be well defined in terms of the fundamental properties $(\varphi)$. Moreover, the dependence relationship between the input data $(\boldsymbol{\alpha})$ and the state variables $(\boldsymbol{\omega})$ may involve severe difficulties. Thus, we symbolically represent the analysis model by means of a system of $n_{\omega}$ implicit equations

$$
\boldsymbol{\psi}(\boldsymbol{\alpha}, \boldsymbol{\omega})=0, \quad \psi(\boldsymbol{\alpha}, \boldsymbol{\omega})=\left\{\psi_{i}(\boldsymbol{\alpha}, \boldsymbol{\omega})\right\}, \quad i=1, \ldots, n_{\omega}
$$

with $n_{\omega}$ unknowns $\left(\boldsymbol{\omega}=\left\{\boldsymbol{\omega}_{i}\right\}\right.$ for $\left.i=1, \ldots, n_{\omega}\right)$, being $\boldsymbol{\psi}(\boldsymbol{\alpha}, \boldsymbol{\omega})=\mathbf{0}$ the socalled state equation. Therefore, the optimum design problem takes the form of a general constrained minimization problem

\begin{tabular}{|ll|}
\hline given & $\boldsymbol{c}$, \\
obtain & $\boldsymbol{x}$, that \\
for & $\boldsymbol{\varphi}=\boldsymbol{\varphi}(\boldsymbol{c}, \boldsymbol{x})$, \\
& $\boldsymbol{\alpha}=\boldsymbol{\alpha}(\boldsymbol{\varphi})$, \\
& $\boldsymbol{\omega}$ such that $\quad \boldsymbol{\psi}(\boldsymbol{\alpha}, \boldsymbol{\omega})=\mathbf{0}$, \\
& $\boldsymbol{\gamma}=\boldsymbol{\gamma}(\boldsymbol{\varphi}, \boldsymbol{\omega})$, \\
minimize & $f(\boldsymbol{\gamma})$, \\
verifying & $g_{j}(\boldsymbol{\gamma}) \leq 0, \quad j=1, \ldots, m$, \\
& $h_{\ell}(\boldsymbol{\gamma})=0, \quad \ell=1, \ldots, p$, \\
&
\end{tabular}

which solution must be found by means of a suitable Mathematical Programming (MP) algorithm [8,10-12]. The set of all possible values of the design variables is called design space. The subset in which the constraints are verified is called feasible region.

\section{SENSITIVITY ANALYSIS}

Mathematical Programming [12] shows that more efficient algorithms can be achieved when the derivatives of the functions that define the problem (objective function and constraints) are known, at least up to the first order. The techniques that let us evaluate these desired derivatives receive the generic name of sensitivity analysis.

Let $\boldsymbol{s}$ be an arbitrary unit vector in the space of design variables, that represents a certain direction in which the actual design is modified. For given 
functions $\boldsymbol{\eta}(\boldsymbol{x})$, and $\boldsymbol{\zeta}(\boldsymbol{\eta})$ the directional derivatives

$$
\begin{aligned}
& D_{\mathrm{s}} \boldsymbol{\eta}(\boldsymbol{x})=\left(\frac{d}{\mathrm{~d} \boldsymbol{x}} \boldsymbol{\eta}(\boldsymbol{x})\right) \boldsymbol{s} \\
& D_{\mathrm{s}}\left(\left.\boldsymbol{\zeta}(\boldsymbol{\eta})\right|_{\boldsymbol{\eta}=\boldsymbol{\eta}(\boldsymbol{x})}\right)=\left.\left(\frac{d}{\mathrm{~d} \boldsymbol{\eta}} \boldsymbol{\zeta}(\boldsymbol{\eta})\right)\right|_{\boldsymbol{\eta}=\boldsymbol{\eta}(\boldsymbol{x})} D_{\mathrm{s}} \boldsymbol{\eta}(\boldsymbol{x})
\end{aligned}
$$

will be abbreviately written as

$$
D_{\mathrm{s}} \boldsymbol{\eta}=\frac{\mathrm{d} \boldsymbol{\eta}}{\mathrm{d} \boldsymbol{x}} \boldsymbol{s}, \quad D_{\mathrm{s}} \boldsymbol{\zeta}=\frac{\mathrm{d} \boldsymbol{\zeta}}{\mathrm{d} \boldsymbol{\eta}} D_{\mathrm{s}} \boldsymbol{\eta} .
$$

In these terms, we shall discuss how to obtain the first order directional derivative $D_{\mathrm{s}} z$ of any given function $z(\boldsymbol{\gamma})$ of the control variables.

\subsection{First Order Direct Differentiation Method}

Direct differentiation of (2) gives the first order direct differentiation method:

$$
\begin{aligned}
\text { given } \quad & \\
\text { obtain } \quad D_{\mathrm{s}} \boldsymbol{\varphi} & =\frac{\partial \boldsymbol{\varphi}}{\partial \boldsymbol{x}} \boldsymbol{s}, \\
D_{\mathrm{s}} \boldsymbol{\alpha} & =\frac{\partial \boldsymbol{\alpha}}{\partial \boldsymbol{\varphi}} D_{\mathrm{s}} \boldsymbol{\varphi}, \\
D_{\mathrm{s}} \boldsymbol{\omega} & \text { such that } \frac{\left.\partial \frac{\partial \boldsymbol{\psi}}{\partial \boldsymbol{\omega}}\right] D_{\mathrm{s}} \boldsymbol{\omega}=-\frac{\partial \boldsymbol{\psi}}{\partial \boldsymbol{\alpha}} D_{\mathrm{s}} \boldsymbol{\alpha}}{\frac{\partial \boldsymbol{\gamma}}{\partial \boldsymbol{\omega}} D_{\mathrm{s}} \boldsymbol{\omega},} \\
D_{\mathrm{s}} \boldsymbol{\gamma} & =\frac{\partial \boldsymbol{\gamma}}{\partial \boldsymbol{\varphi}} D_{\mathrm{s}} \boldsymbol{\varphi} \\
D_{\mathrm{s}} z & =\frac{d z}{\mathrm{~d} \boldsymbol{\gamma}} D_{\mathrm{s}} \boldsymbol{\gamma},
\end{aligned}
$$

where obtaining the directional derivatives of the state variables requires solving the linear system of $n_{\omega}$ equations with $n_{\omega}$ unknowns

$$
\left[\frac{\partial \boldsymbol{\psi}}{\partial \boldsymbol{\omega}}\right] D_{\mathrm{s}} \boldsymbol{\omega}=-\frac{\partial \boldsymbol{\psi}}{\partial \boldsymbol{\alpha}} D_{\mathrm{s}} \boldsymbol{\alpha}
$$

In engineering practice, equation (1) is frequently a discretized form of a certain boundary-value problem. Most of the available analysis programs to solve 
this kind of problems (i.e. wide purpose FEM or BEM codes) do not provide the derivatives of the state function $([\partial \boldsymbol{\psi} / \partial \boldsymbol{\omega}]$ and $\partial \boldsymbol{\psi} / \partial \boldsymbol{\alpha})$ required by $(6)$. In this cases, only finite difference approximations can be used to obtain the directional derivatives of the state variables. This produces a significative loss of accuracy in the information supplied to the Mathematical Programming algorithm and a high computational cost $[1,7]$. On the other hand, to implement the additional computations required by (6) may involve some unexpected conceptual and practical difficulties, depending on the particular form of the state equation that describes the underlying physical phenomena, and on the numerical strategy outlined to solve it.

In optimum structural design — and particularly when the structural analysis is performed by a FEM code- explicit distinctions are made between sizing (fixed-geometry) optimization and shape optimization $[1,13]$. In the former, the difficulties involved in the differentiation of the state equation are significantly reduced, provided that the input variables that define the structural shape do not depend on the design variables, but only on the design constants. In the latter, some subtle aspects - related to the differentiation of functions defined by integration in variable domains - interfere in the sensitivity analysis.

To these topics is mainly devoted this paper.

\subsection{Higher order derivatives}

For a given set of unit vectors $\left\{\boldsymbol{s}_{\kappa}\right\}, \kappa=1, \ldots, k$, we are interested in computing

$$
D_{\mathrm{s}_{1}, \mathrm{~s}_{2}, \ldots, \mathrm{s}_{\mathrm{k}}} z=D_{\mathrm{s}_{\mathrm{k}}}\left(\ldots D_{\mathrm{s}_{2}}\left(D_{\mathrm{s}_{1}} z\right)\right)
$$

A scheme for high order sensitivity analysis can be easily derived following the same principles outlined before. Conceptually, such a scheme is only slightly more complex than the first order one, but the computational requirements increase exponentially with the order of differentiation, due to the number of derivatives to be computed [8,9]. This precludes the use of Mathematical Programming algorithms that require full high order information. However, a high order sensitivity analysis for a given direction in the design space can be performed with relatively small computational requirements. This provides an extremely useful tool for improving first order algorithms $[6,8,10,11]$. 


\subsection{Adjoint State}

It is easy to show how the direct differentiation computational scheme (5) can be reordered [9], giving the so-called adjoint state method

$$
\begin{aligned}
& \text { given: } \boldsymbol{s}, \\
& \text { obtain: } \quad D_{\mathrm{s}} \boldsymbol{\varphi}=\frac{\partial \boldsymbol{\varphi}}{\partial \boldsymbol{x}} \boldsymbol{s}, \\
& D_{\mathrm{s}} \boldsymbol{\alpha}=\frac{\partial \boldsymbol{\alpha}}{\partial \boldsymbol{\varphi}} D_{\mathrm{s}} \boldsymbol{\varphi}, \\
& \boldsymbol{\mu}_{z} \quad \text { such that } \\
& D_{\mathrm{s}} z\left.=\frac{d z}{\mathrm{~d} \boldsymbol{\gamma}} \frac{\partial \boldsymbol{\gamma}}{\partial \boldsymbol{\varphi}}\right]^{T} D_{\mathrm{s}} \boldsymbol{\boldsymbol { \mu } _ { z }}=-\left(\frac{d z}{\mathrm{~d} \boldsymbol{\gamma}} \frac{\partial \boldsymbol{\gamma}}{\partial \boldsymbol{\omega}}\right)^{T} \\
&
\end{aligned}
$$

where the unknown vector $\mu_{z}$ is known as the adjoint state corresponding to the function $z(\boldsymbol{\gamma})$, associated to the so-called direct state (5). While in (5) it is necessary to compute the derivatives of the state variables $\left(D_{\mathrm{s}} \boldsymbol{\omega}\right)$ as an intermediate result for each direction $\boldsymbol{s}$, in (8) it is necessary to compute $\boldsymbol{\mu}_{z}$ for each function $z(\boldsymbol{\gamma})$. Therefore, (8) will be preferred rather than (5) when the number of functions to be derived is significantly smaller than the number of directions in which derivatives must be computed [14].

Normally, the adjoint state scheme in design optimization does not offer significant advantages over the direct differentiation scheme. Consider that in practical optimization problems, the number of constraints is often much larger than the number of design variables. In any case, a wide purpose general optimum design system must include the possibility of using any of both schemes, depending on the problem statement.

\section{SENSITIVITY ANALYSIS FOR INTEGRAL METHODS}

\subsection{Discretized State Equations in Integral Methods}

As it was mentioned before, equation (1) is frequently a discretized form obtained by means of one of the so-called integral methods - of a certain boundary-value problem. 
Let the original form of this problem be:

$$
\begin{array}{lll}
\text { find } & u(\boldsymbol{r}, \boldsymbol{\alpha}), & \boldsymbol{r} \in \bar{\Omega}(\boldsymbol{\alpha}) \subset \mathbb{R}^{\operatorname{dim}(\Omega)} \\
\text { such that } & \mathcal{P}(u, \boldsymbol{\alpha})=0 & \text { on } \Omega(\boldsymbol{\alpha}) \\
\text { and } & \mathcal{B}(u, \boldsymbol{\alpha})=0 & \text { on } \Gamma(\boldsymbol{\alpha}) \subset \bar{\Omega}(\boldsymbol{\alpha})
\end{array}
$$

where $\Omega$ is an open bounded domain with Lipschitzian boundary $\partial \Omega$ and closure $\bar{\Omega}, \bar{\Gamma}$ is the subset of $\bar{\Omega}$ where (boundary) conditions are prescribed, $\boldsymbol{r}$ is the material coordinates vector of an arbitrary point in $\bar{\Omega}$, and $\mathcal{P}$ and $\mathcal{B}$ are generic differential-algebraic operators that represent the system of partial differential equations that must be satisfied on $\Omega$ and the prescribed conditions that must be fulfilled on $\Gamma$.

In these terms, the state equation (1) is generally derived as follows:

(i) First, the so-called strong — or classical — form of the problem (9) must be reduced to an equivalent weak - or variational - form. If this process is based on a weighted residual approach [15,16], as usual, the variational form can be written as

$$
\begin{aligned}
& \left.\int_{\boldsymbol{r} \in \Omega(\boldsymbol{\alpha})} \mathcal{P}(u, \boldsymbol{\alpha})\right|_{u=u(\boldsymbol{r}, \boldsymbol{\alpha})} \varpi(\boldsymbol{r}, \boldsymbol{\alpha}) \mathrm{d} \Omega+ \\
& \left.\int_{\boldsymbol{r} \in \Gamma(\boldsymbol{\alpha})} \mathcal{B}(u, \boldsymbol{\alpha})\right|_{u=u(\boldsymbol{r}, \boldsymbol{\alpha})} \varpi(\boldsymbol{r}, \boldsymbol{\alpha}) \mathrm{d} \Gamma=0,
\end{aligned}
$$

that must hold for all members $\varpi(\boldsymbol{r}, \boldsymbol{\alpha})$ of a suitable class $\mathcal{H}_{\varpi}$ of test functions defined on $\bar{\Omega}(\boldsymbol{\alpha})$. The actual expression of the variational form may differ from the one exposed in (10), since additional analytical work (i.e. application of Greens' Identity [17], integration by parts or the Divergence theorem [15]) could be specifically applied, as the case may require, in order to get more compact expressions, reduce derivability requisites, etc.

(ii) Let $\mathcal{H}_{u}$ be the class of trial functions (candidate solutions to satisfy the above stated variational form), and let $u(\boldsymbol{r}, \boldsymbol{\alpha}) \in \mathcal{H}_{u}$ be the exact solution of the previous problem. The first step to develop the method is to construct a finite-dimensional (discretized) approximation [15] of $\mathcal{H}_{u}$. Thus, for a chosen set of trial functions

$$
\boldsymbol{\nu}(\boldsymbol{r}, \boldsymbol{\alpha})=\left\{\nu_{i}(\boldsymbol{r}, \boldsymbol{\alpha})\right\}, \quad i=1, \ldots, n_{\omega}
$$

let $\mathcal{H}_{u}^{\nu} \subset \mathcal{H}_{u}$ be the collection of functions

$$
\widehat{u}(\boldsymbol{r}, \boldsymbol{\alpha}, \boldsymbol{\omega})=\sum_{i=1}^{n_{\omega}} \omega_{i} \nu_{i}(\boldsymbol{r}, \boldsymbol{\alpha})=\boldsymbol{\omega}^{T} \boldsymbol{\nu}(\boldsymbol{r}, \boldsymbol{\alpha}) .
$$

The objective is to approximate the exact solution $u(\boldsymbol{r}, \boldsymbol{\alpha})$ of $(10)$ in this finite-dimensional context. Namely, for given values of the input variables 
$\boldsymbol{\alpha}$ of the model, the unknown values of the state variables $\boldsymbol{\omega}$ must be determined (1) in such a way that the corresponding discretized solution (12) is as close as possible to the exact solution of the boundary-value problem.

(iii) Since the exact solution $u(r, \boldsymbol{\alpha})$ will not be included (as a general rule) in the subspace $\mathcal{H}_{u}^{\boldsymbol{\nu}}$, variational equality (10) will not hold anymore. However, if we restrict the class of test functions $\mathcal{H}_{\varpi}$ to the collection $\mathcal{H}_{\varpi}^{\boldsymbol{\pi}}$ generated by a chosen set of test functions

$$
\boldsymbol{\pi}(\boldsymbol{r}, \boldsymbol{\alpha})=\left\{\pi_{i}(\boldsymbol{r}, \boldsymbol{\alpha})\right\}, \quad i=1, \ldots, n_{\omega}
$$

the variational equality (10) is reduced to the system of $n_{\omega}$ equations

$$
\begin{aligned}
& \left.\int_{\boldsymbol{r} \in \Omega(\boldsymbol{\alpha})} \mathcal{P}(u, \boldsymbol{\alpha})\right|_{u=\widehat{u}(\boldsymbol{r}, \boldsymbol{\alpha}, \boldsymbol{\omega})} \pi_{i}(\boldsymbol{r}, \boldsymbol{\alpha}) d \Omega+ \\
& \left.\int_{\boldsymbol{r} \in \Gamma(\boldsymbol{\alpha})} \mathcal{B}(u, \boldsymbol{\alpha})\right|_{u=\widehat{u}(\boldsymbol{r}, \boldsymbol{\alpha}, \boldsymbol{\omega})} \pi_{i}(\boldsymbol{r}, \boldsymbol{\alpha}) d \Gamma=0, \quad i=1, \ldots, n_{\omega},
\end{aligned}
$$

with $n_{\omega}$ unknowns $\boldsymbol{\omega}$.

(iv) For practical reasons, domains $\bar{\Omega}$ and $\bar{\Gamma}$ can be discretized in subdomains $[18,19]$. Thus

$$
\bar{\Omega}(\boldsymbol{\alpha})=\bigcup_{e=1}^{n_{\Omega}} \bar{\Omega}_{e}(\boldsymbol{\alpha}) \quad \text { and } \quad \bar{\Gamma}(\boldsymbol{\alpha})=\bigcup_{b=1}^{n_{\Gamma}} \bar{\Gamma}_{b}(\boldsymbol{\alpha})
$$

where each subdomain $\left(\bar{\Omega}_{e}\right.$ or $\left.\bar{\Gamma}_{b}\right)$ is closed and consist on a nonempty interior $\left(\Omega_{e}\right.$ or $\Gamma_{b}$, respectively) and a Lipschitzian boundary $\left(\partial \Omega_{e}\right.$ or $\partial \Gamma_{b}$, respectively), and

$$
\begin{aligned}
\Omega_{e_{1}}(\boldsymbol{\alpha}) \bigcap \Omega_{e_{2}}(\boldsymbol{\alpha})=\emptyset & \forall e_{1} \neq e_{2}, \\
\Gamma_{b_{1}}(\boldsymbol{\alpha}) \bigcap \Gamma_{b_{2}}(\boldsymbol{\alpha})=\emptyset & \forall b_{1} \neq b_{2}
\end{aligned}
$$

(v) And, finally, equations (14) can be reduced to the standard form (1), being

$$
\boldsymbol{\psi}(\boldsymbol{\alpha}, \boldsymbol{\omega})=\sum_{e=1}^{n_{\Omega}} \boldsymbol{\psi}_{e}^{\Omega}(\boldsymbol{\alpha}, \boldsymbol{\omega})+\sum_{b=1}^{n_{\Gamma}} \boldsymbol{\psi}_{b}^{\Gamma}(\boldsymbol{\alpha}, \boldsymbol{\omega})=0
$$

with

$$
\begin{aligned}
\boldsymbol{\psi}_{e}^{\Omega}(\boldsymbol{\alpha}, \boldsymbol{\omega}) & =\left.\int_{\boldsymbol{r} \in \Omega_{e}(\boldsymbol{\alpha})} \mathcal{P}(u, \boldsymbol{\alpha})\right|_{u=\widehat{u}(\boldsymbol{r}, \boldsymbol{\alpha}, \boldsymbol{\omega})} \pi(\boldsymbol{r}, \boldsymbol{\alpha}) \mathrm{d} \Omega \\
\boldsymbol{\psi}_{b}^{\Gamma}(\boldsymbol{\alpha}, \boldsymbol{\omega}) & =\left.\int_{\boldsymbol{r} \in \Gamma_{b}(\boldsymbol{\alpha})} \mathcal{B}(u, \boldsymbol{\alpha})\right|_{u=\widehat{u}(\boldsymbol{r}, \boldsymbol{\alpha}, \boldsymbol{\omega})} \boldsymbol{\pi}(\boldsymbol{r}, \boldsymbol{\alpha}) \mathrm{d} \Gamma
\end{aligned}
$$

Thus, contributions $\boldsymbol{\psi}_{e}^{\Omega}$ and $\boldsymbol{\psi}_{b}^{\Gamma}$ to the state equation (1) are obtained by 

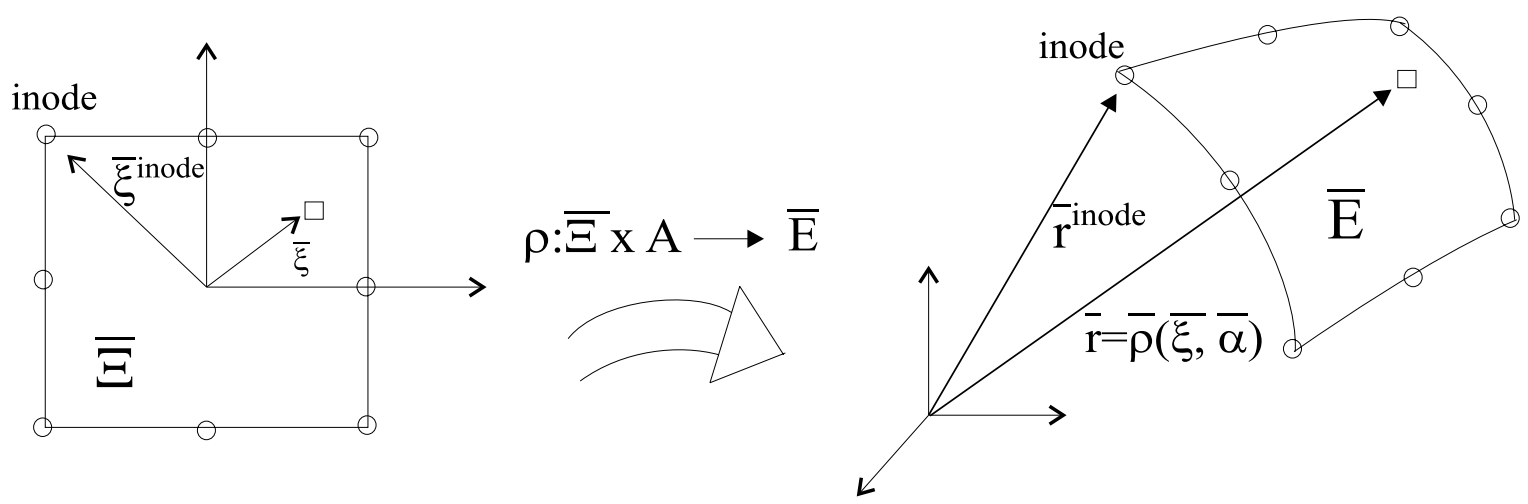

Fig. 1. Standard FEM Mapping

computing and assembling terms of the general type

$$
\boldsymbol{\psi}^{E}(\boldsymbol{\alpha}, \boldsymbol{\omega})=\int_{\boldsymbol{r} \in E(\boldsymbol{\alpha})} \boldsymbol{\phi}^{E}(\boldsymbol{r}, \boldsymbol{\alpha}, \boldsymbol{\omega}) \mathrm{d} E
$$

where $\bar{E}(\boldsymbol{\alpha})$ is an element (that is, a closed subdomain with nonempty interior $E(\boldsymbol{\alpha})$ and Lipschitzian boundary $\partial E(\boldsymbol{\alpha}))$ of $\operatorname{dimension} \operatorname{dim}(E) \leq \operatorname{dim}(\Omega)$ within domain $\bar{\Omega}$.

\subsection{Standard definition of elements}

It is obvious that trying to calculate the element contributions (19) in terms of the material coordinates $\boldsymbol{r}$ would be awkward [18,19]. However, in most of the cases it is relatively easy to introduce an invertible differentiable mapping (see Figure 1)

$$
\begin{array}{cl}
\boldsymbol{\rho}: \bar{\Xi} \times A & \bar{\Omega} \\
(\boldsymbol{\xi}, \boldsymbol{\alpha}) & \boldsymbol{r}=\boldsymbol{\rho}(\boldsymbol{\xi}, \boldsymbol{\alpha})
\end{array}
$$

such that the element $\bar{E}(\boldsymbol{\alpha})=\boldsymbol{\rho}(\bar{\Xi}, \boldsymbol{\alpha})$ is the image of a convenient fixed reference domain $\Xi$ (also called master element or parent domain) by the coordinate transformation $\boldsymbol{\rho}$. Then, every point in the element $\bar{E}$, given by its material (also called global) coordinates

$$
\boldsymbol{r}=\left\{r_{i}\right\}, \quad i=1, \ldots, n_{r}=\operatorname{dim}(\Omega)
$$

is the image of an unique corresponding point in the reference domain $\bar{\Xi}$, given by its reference (also called local or natural) coordinates

$$
\boldsymbol{\xi}=\left\{\xi_{i}\right\}, \quad i=1, \ldots, n_{\xi}=\operatorname{dim}(E),
$$


and the mapping depends on the input variables $\boldsymbol{\alpha}$ that describe the numerical model.

In a FEM context such a transformation is inherent to the formulation, and is normally written as

$$
\boldsymbol{\rho}(\boldsymbol{\xi}, \boldsymbol{\alpha})=\sum_{\text {inode }=1}^{\text {nnode }} \boldsymbol{r}^{\text {inode }}(\boldsymbol{\alpha}) N^{\text {inode }}(\boldsymbol{\xi})
$$

where the master element $\Xi$ is defined by the reference coordinates $\left\{\boldsymbol{\xi}^{\text {inode }}\right\}$ of the "nnode" so-called nodal points (or nodes) of the element [15,20]. In these terms, the element $\bar{E}$ is defined by the corresponding material coordinates of the nodal points $\left\{\boldsymbol{r}^{\text {inode }}(\boldsymbol{\alpha})\right\}$, and the so-called shape functions

$$
\begin{array}{cl}
N^{\text {inode }}: \bar{\Xi} \longrightarrow & \mathbb{R} \\
\boldsymbol{\xi} & N^{\text {inode }}(\boldsymbol{\xi})
\end{array}
$$

that must verify the standard interpolation conditions

$$
N^{\text {inode }}\left(\xi^{\text {jnode }}\right)= \begin{cases}0, & \text { if inode } \neq \text { jnode } \\ 1, & \text { otherwise }\end{cases}
$$

In these terms, the jacobian matrix of the transformation (20) can be written as

$$
\boldsymbol{J}(\boldsymbol{\xi}, \boldsymbol{\alpha})=\frac{\partial \boldsymbol{\rho}(\xi, \boldsymbol{\alpha})}{\partial \boldsymbol{\xi}}=\sum_{\text {inode }=1}^{\text {nnode }} \boldsymbol{r}^{\text {inode }}(\boldsymbol{\alpha}) \frac{\partial}{\partial \boldsymbol{\xi}} N^{\text {inode }}(\boldsymbol{\xi})
$$

Now, it seems clear that contributions (19) should be computed by integration in terms of the reference coordinates $\boldsymbol{\xi}$. Thus (19) must be reduced to the standard form

$$
\boldsymbol{\psi}^{E}(\boldsymbol{\alpha}, \boldsymbol{\omega})=\int_{\boldsymbol{\xi} \in \Xi} \boldsymbol{\phi}^{\Xi}(\boldsymbol{\xi}, \boldsymbol{\alpha}, \boldsymbol{\omega})\left|\frac{\mathrm{d} E}{\mathrm{~d} \Xi}\right| \mathrm{d} \Xi, \quad \mathrm{d} \Xi=\prod_{i=1}^{n_{\xi}} \mathrm{d} \xi_{i}
$$

It is obvious that

$$
\boldsymbol{\phi}^{\Xi}(\boldsymbol{\xi}, \boldsymbol{\alpha}, \boldsymbol{\omega})=\left.\boldsymbol{\phi}^{E}(\boldsymbol{r}, \boldsymbol{\alpha}, \boldsymbol{\omega})\right|_{\boldsymbol{r}=\boldsymbol{\rho}(\xi, \boldsymbol{\alpha})} .
$$

On the other hand, it is widely known [21] that for $n_{\xi}=n_{r}$ the integration jacobian $|\mathrm{d} E / \mathrm{d} \Xi|$ is the determinant of the jacobian matrix (26). This result 
is frequently referred to as Theorem of Gauss-Binnet. Otherwise, the value $|\mathrm{d} E / \mathrm{d} \Xi|$ is generally computed by means of a specific expression that depends on the dimension $n_{r}$ of the material coordinates space and the dimension $n_{\xi}$ of the reference coordinates space. In engineering practice $n_{r}=3$ as maximum. Thus, when $n_{\xi}=1, E$ is a curve and $|\mathrm{d} E / \mathrm{d} \Xi|$ is normally computed as the modulus of the tangent vector; on the other hand, when $n_{\xi}=2, E$ is a surface and $|\mathrm{d} E / \mathrm{d} \Xi|$ is normally computed as the modulus of the normal vector.

\subsection{Integration of element contributions in reference coordinates}

It seems to be not so widely known that the value $|\mathrm{d} E / \mathrm{d} \Xi|$ in $(27)$, that is the integration jacobian corresponding to the mapping (20), admits the following expression

$$
\mathrm{d} E=\left|\frac{\mathrm{d} E}{\mathrm{~d} \Xi}\right| \mathrm{d} \Xi=\sqrt{\operatorname{det}[\boldsymbol{G}(\boldsymbol{\xi}, \boldsymbol{\alpha})]} \mathrm{d} \Xi, \quad \mathrm{d} \Xi=\prod_{i=1}^{n_{\xi}} \mathrm{d} \xi_{i},
$$

where

$$
\boldsymbol{G}(\boldsymbol{\xi}, \boldsymbol{\alpha})=\boldsymbol{J}^{T}(\boldsymbol{\xi}, \boldsymbol{\alpha}) \boldsymbol{J}(\boldsymbol{\xi}, \boldsymbol{\alpha})
$$

is the so-called metric tensor [22] of the riemannian manifold $E(\boldsymbol{\alpha})$ (see Appendix I). An original, comprehensive and straightforward proof of (29) is given in Appendix I. A classical, more involved proof can be found in [23]. This expression gives the " $n_{\xi}$-dimensional hypersurface element in $\mathbb{R}^{n_{r}}$ ", being $\mathrm{d} E$ the generalized volume of the $n_{\xi}$-dimensional hypercube defined by the infinitesimal vectors $\left\{\boldsymbol{t}_{i}(\boldsymbol{\xi}, \boldsymbol{\alpha}) \mathrm{d} \xi_{i}\right\}$, where $\boldsymbol{t}_{i}(\boldsymbol{\xi}, \boldsymbol{\alpha})=\partial \boldsymbol{\rho}(\boldsymbol{\xi}, \boldsymbol{\alpha}) / \partial \xi_{i}$ for $i=1, \ldots, n_{\xi}$ are the so-called natural vectors of the reference coordinates. Thus, the integration jacobian $|\mathrm{d} E / \mathrm{d} \Xi|$ is the square root of the determinant of the metric tensor.

It is interesting to notice that this expression for the integration jacobian is intrinsic to the riemannian $n_{\xi}$-dimensional manifold. Thus, once the metric tensor is known, the integration jacobian is completely defined regardless of the dimension $n_{r}$ of the material space and the specific mapping (20) that we use. Obviously, (29) is equivalent to the usual expressions of the differential elements of arc length, surface and volume when $n_{r} \leq 3$.

Hence, contributions (19) can be computed as

$$
\boldsymbol{\psi}^{E}(\boldsymbol{\alpha}, \boldsymbol{\omega})=\int_{\boldsymbol{\xi} \in \Xi} \boldsymbol{\phi}^{\Xi}(\boldsymbol{\xi}, \boldsymbol{\alpha}, \boldsymbol{\omega}) \sqrt{\operatorname{det}[\boldsymbol{G}(\boldsymbol{\xi}, \boldsymbol{\alpha})]} \mathrm{d} \Xi
$$


being this expression valid for all cases $n_{\xi} \leq n_{r}$. Obviously, the metric tensor is required to be positive-definite, for the mapping (23) to be acceptable (see Appendix I). Therefore, det $[\boldsymbol{G}(\boldsymbol{\xi}, \boldsymbol{\alpha})]>0$ and the integration jacobian in (31) is always well defined.

Finally, a numerical quadrature (very often a Gauss type formula) could be implemented, resulting in

$$
\boldsymbol{\psi}^{E}(\boldsymbol{\alpha}, \boldsymbol{\omega}) \approx \sum_{\text {igaue }=1}^{\text {ngaue }} \boldsymbol{\phi}^{\Xi}\left(\boldsymbol{\xi}^{\text {igaue }}, \boldsymbol{\alpha}, \boldsymbol{\omega}\right) \sqrt{\operatorname{det}\left[\boldsymbol{G}\left(\boldsymbol{\xi}^{\text {igaue }}, \boldsymbol{\alpha}\right)\right]} W^{\text {igaue }}
$$

for selected sets of "ngaue"integration points $\left\{\boldsymbol{\xi}^{\text {igaue }}\right\}$ and weights $\left\{W^{\text {igaue }}\right\}$.

The above stated numerical integration procedure does not depend either on the dimension of the material coordinates space $\left[n_{r}\right]$ nor on the dimension of the reference coordinates space $\left[n_{\xi}\right]$. Thus, a general purpose subroutine should be able to compute contributions (19) independently of the dimension of the problem - generally 1D, 2D or 3D (it could be higher in special applications) and of the dimension of the elements being used.

\subsection{First Order Sensitivity Analysis in Integral Methods}

At this point we recall equations (5). For a given arbitrary unit vector $\boldsymbol{s}$ in the space of design variables one should easily compute the directional derivative of the input variables $\left(D_{\mathrm{s}} \boldsymbol{\alpha}\right)$. Therefore, taking into account equations (17), one concludes that the terms which computation we must discuss are

$$
\left[\frac{\partial \boldsymbol{\psi}}{\partial \boldsymbol{\omega}}\right]=\sum_{e=1}^{n_{\Omega}}\left[\frac{\partial}{\partial \boldsymbol{\omega}} \boldsymbol{\psi}_{e}^{\Omega}(\boldsymbol{\alpha}, \boldsymbol{\omega})\right]+\sum_{b=1}^{n_{\Gamma}}\left[\frac{\partial}{\partial \boldsymbol{\omega}} \boldsymbol{\psi}_{b}^{\Gamma}(\boldsymbol{\alpha}, \boldsymbol{\omega})\right]
$$

and

$$
D_{\mathrm{s}}^{\boldsymbol{\alpha}} \boldsymbol{\psi}=\sum_{e=1}^{n_{\Omega}} D_{\mathrm{s}}^{\boldsymbol{\alpha}} \boldsymbol{\psi}_{e}^{\Omega}(\boldsymbol{\alpha}, \boldsymbol{\omega})+\sum_{b=1}^{n_{\Gamma}} D_{\mathrm{s}}^{\boldsymbol{\alpha}} \psi_{b}^{\Gamma}(\boldsymbol{\alpha}, \boldsymbol{\omega})
$$

where we introduce the symbolic operator

$$
D_{\mathrm{s}}^{\boldsymbol{\alpha}} \square=\frac{\partial \square}{\partial \boldsymbol{\alpha}} D_{\mathrm{s}} \boldsymbol{\alpha}
$$


In these terms, (19) shows that the derivatives of the contributions $\boldsymbol{\psi}_{e}^{\Omega}$ and $\boldsymbol{\psi}_{b}^{\Gamma}$ should be obtained by computing and assembling terms of the general type

$$
\left[\frac{\partial}{\partial \boldsymbol{\omega}} \boldsymbol{\psi}^{E}(\boldsymbol{\alpha}, \boldsymbol{\omega})\right]=\left[\frac{\partial}{\partial \boldsymbol{\omega}}\left(\int_{\boldsymbol{r} \in E(\boldsymbol{\alpha})} \boldsymbol{\phi}^{E}(\boldsymbol{r}, \boldsymbol{\alpha}, \boldsymbol{\omega}) \mathrm{d} E\right)\right],
$$

and

$$
D_{\mathrm{s}}^{\boldsymbol{\alpha}} \boldsymbol{\psi}^{E}(\boldsymbol{\alpha}, \boldsymbol{\omega})=D_{\mathrm{s}}^{\boldsymbol{\alpha}}\left(\int_{\boldsymbol{r} \in E(\boldsymbol{\alpha})} \boldsymbol{\phi}^{E}(\boldsymbol{r}, \boldsymbol{\alpha}, \boldsymbol{\omega}) \mathrm{d} E\right)
$$

Normally, the shape of elements does not depend on the state variables $\omega$. Thus, computation of terms (36) is considered trivial since

$$
\left[\frac{\partial}{\partial \boldsymbol{\omega}} \boldsymbol{\psi}^{E}(\boldsymbol{\alpha}, \boldsymbol{\omega})\right]=\left[\int_{\boldsymbol{r} \in E(\boldsymbol{\alpha})} \frac{\partial}{\partial \boldsymbol{\omega}} \boldsymbol{\phi}^{E}(\boldsymbol{r}, \boldsymbol{\alpha}, \boldsymbol{\omega}) \mathrm{d} E\right]
$$

which can be computed by integration in reference coordinates as

$$
\left[\frac{\partial}{\partial \boldsymbol{\omega}} \psi^{E}(\boldsymbol{\alpha}, \boldsymbol{\omega})\right]=\left[\int_{\boldsymbol{\xi} \in \Xi} \frac{\partial}{\partial \boldsymbol{\omega}} \boldsymbol{\phi}^{\Xi}(\boldsymbol{\xi}, \boldsymbol{\alpha}, \boldsymbol{\omega}) \sqrt{\operatorname{det}[\boldsymbol{G}(\boldsymbol{\xi}, \boldsymbol{\alpha})]} \mathrm{d} \Xi\right]
$$

In shape optimization, however, the shape of elements does depend on the input variables $\boldsymbol{\alpha}$. For this reason, computation of terms (37) is much more difficult, since the integration domains vary. Therefore, the derivative of the integral cannot be computed by integration of the derivative, as in the former case.

\subsection{First Order Shape Sensitivity}

Our goal is to compute contributions (37). Using equation (31) we can write

$$
D_{\mathrm{s}}^{\boldsymbol{\alpha}} \boldsymbol{\psi}^{E}(\boldsymbol{\alpha}, \boldsymbol{\omega})=\int_{\boldsymbol{\xi} \in \Xi} D_{\mathrm{s}}^{\boldsymbol{\alpha}}\left(\boldsymbol{\phi}^{\Xi}(\boldsymbol{\xi}, \boldsymbol{\alpha}, \boldsymbol{\omega}) \sqrt{\operatorname{det}[G(\boldsymbol{\xi}, \boldsymbol{\alpha})]}\right) d \Xi .
$$

For the sake of compacity we define the symbolic operator $\mathcal{D}_{\mathrm{s}}^{\Xi}$ such that

$$
\mathcal{D}_{\mathrm{s}}^{\Xi} \square=D_{\mathrm{s}}^{\boldsymbol{\alpha}} \square+\square \frac{1}{2} D_{\mathrm{s}}^{\boldsymbol{\alpha}} \ln (\operatorname{det}[\boldsymbol{G}(\boldsymbol{\xi}, \boldsymbol{\alpha})])
$$


which reduces $(40)$ to the compact form

$$
D_{\mathrm{s}}^{\boldsymbol{\alpha}} \boldsymbol{\psi}^{E}(\boldsymbol{\alpha}, \boldsymbol{\omega})=\int_{\boldsymbol{\xi} \in \Xi} \mathcal{D}_{\mathrm{s}}^{\Xi}\left(\boldsymbol{\phi}^{\Xi}(\boldsymbol{\xi}, \boldsymbol{\alpha}, \boldsymbol{\omega})\right) \sqrt{\operatorname{det}[\boldsymbol{G}(\boldsymbol{\xi}, \boldsymbol{\alpha})]} \mathrm{d} \Xi .
$$

Finally, by means of some additional analytical work [24] we obtain the explicit ready-for-computation expression

$$
\mathcal{D}_{\mathrm{s}}^{\Xi} \square=D_{\mathrm{s}}^{\boldsymbol{\alpha}} \square+\square \frac{1}{2} \operatorname{Tr}\left[\boldsymbol{G}^{-1}(\boldsymbol{\xi}, \boldsymbol{\alpha}) D_{\mathrm{s}}^{\boldsymbol{\alpha}} \boldsymbol{G}(\boldsymbol{\xi}, \boldsymbol{\alpha})\right]
$$

where direct differentiation of (30) and (26) gives

$$
D_{\mathrm{s}}^{\boldsymbol{\alpha}} \boldsymbol{G}(\boldsymbol{\xi}, \boldsymbol{\alpha})=\left[\boldsymbol{J}^{T}(\boldsymbol{\xi}, \boldsymbol{\alpha}) D_{\mathrm{s}}^{\boldsymbol{\alpha}} \boldsymbol{J}(\boldsymbol{\xi}, \boldsymbol{\alpha})\right]+\left[\boldsymbol{J}^{T}(\boldsymbol{\xi}, \boldsymbol{\alpha}) D_{\mathrm{s}}^{\boldsymbol{\alpha}} \boldsymbol{J}(\boldsymbol{\xi}, \boldsymbol{\alpha})\right]^{T}
$$

and

$$
D_{\mathrm{s}}^{\boldsymbol{\alpha}} \boldsymbol{J}(\boldsymbol{\xi}, \boldsymbol{\alpha})=\sum_{\text {inode=1 }}^{\text {nnode }} D_{\mathrm{s}}^{\boldsymbol{\alpha}} \boldsymbol{r}^{\text {inode }}(\boldsymbol{\alpha}) \frac{\partial}{\partial \boldsymbol{\xi}} N^{\text {inode }}(\boldsymbol{\xi})
$$

On the other hand, (42) can be written in terms of the material coordinates as

$$
D_{\mathrm{s}}^{\boldsymbol{\alpha}} \boldsymbol{\psi}^{E}(\boldsymbol{\alpha}, \boldsymbol{\omega})=\int_{\boldsymbol{r} \in E(\boldsymbol{\alpha})} \mathcal{D}_{\mathrm{s}}^{E} \boldsymbol{\phi}^{E}(\boldsymbol{r}, \boldsymbol{\alpha}, \boldsymbol{\omega}) \mathrm{d} E
$$

by means of the corresponding symbolic operator

$$
\mathcal{D}_{\mathrm{s}}^{E} \square=\left.\mathcal{D}_{\mathrm{s}}^{\Xi}\left(\left.\square\right|_{\boldsymbol{r}=\rho(\xi, \alpha)}\right)\right|_{\boldsymbol{\xi}=\boldsymbol{\rho}^{-1}(\boldsymbol{r}, \boldsymbol{\alpha})} .
$$

Expressions (42) and (46) are equivalent. So are operators (43) and (47). Since integration is performed in reference coordinates, the expression (42) and the operator (43) will be preferred in practice. However, equation (46) shows that the derivative of an integral with respect to a parameter that modifies the integration domain can be easily calculated as the integral of the operator (47) applied to the subintegrand function, that is

$$
D_{\mathrm{s}}^{\boldsymbol{\alpha}}\left(\int_{\boldsymbol{r} \in E(\boldsymbol{\alpha})} \boldsymbol{\phi}^{E}(\boldsymbol{r}, \boldsymbol{\alpha}, \boldsymbol{\omega}) \mathrm{d} E\right)=\int_{\boldsymbol{r} \in E(\boldsymbol{\alpha})} \mathcal{D}_{\mathrm{s}}^{E} \boldsymbol{\phi}^{E}(\boldsymbol{r}, \boldsymbol{\alpha}, \boldsymbol{\omega}) \mathrm{d} E,
$$


which explains why recurrency is allowed in high order shape sensitivity expressions.

\subsection{High Order Shape Sensitivity}

When a shape sensitivity analysis of order $k>1$ is required, we must develop symbolic terms of the general type

$$
\begin{aligned}
& D_{\mathrm{s}_{\kappa_{\alpha}}^{\alpha}}^{\boldsymbol{\alpha}} \cdots D_{\mathrm{s}_{1}^{\alpha}}^{\boldsymbol{\alpha}} D_{\mathrm{s}_{\kappa_{\omega}}^{\omega}}^{\boldsymbol{\omega}} \cdots D_{\mathrm{s}_{1}^{\omega}}^{\boldsymbol{\omega}} \boldsymbol{\psi}^{E}= \\
& D_{\mathrm{s}_{\kappa \alpha}^{\alpha}}^{\boldsymbol{\alpha}} \cdots D_{\mathrm{s}_{1}^{\alpha}}^{\boldsymbol{\alpha}} D_{\mathrm{s}_{\kappa \omega}^{\omega}}^{\boldsymbol{\omega}} \cdots D_{\mathrm{s}_{1}^{\omega}}^{\boldsymbol{\omega}}\left(\int_{\boldsymbol{r} \in E(\boldsymbol{\alpha})} \boldsymbol{\phi}^{E}(\boldsymbol{r}, \boldsymbol{\alpha}, \boldsymbol{\omega}) \mathrm{d} E\right)
\end{aligned}
$$

for values $0 \leq \kappa_{\alpha} \leq k, 0 \leq \kappa_{\omega} \leq k, 1 \leq \kappa_{\alpha}+\kappa_{\omega} \leq k$. Obviously, all the related directional derivatives of lower order (up to the order $k-1$ ) of the state variables, along directions $\boldsymbol{s}_{1}^{\alpha}, \ldots, \boldsymbol{s}_{\kappa_{\alpha}}^{\alpha}$ and $\boldsymbol{s}_{1}^{\omega}, \ldots, \boldsymbol{s}_{\kappa_{\omega}}^{\omega}$ should be computed in advance. In (49) we use the symbolic operators.

$$
D_{\mathrm{s}}^{\boldsymbol{\alpha}} \square=\frac{\partial \square}{\partial \boldsymbol{\alpha}} D_{\mathrm{s}} \boldsymbol{\alpha}, \quad D_{\mathrm{s}}^{\boldsymbol{\omega}} \square=\frac{\partial \square}{\partial \boldsymbol{\omega}} D_{\mathrm{s}} \boldsymbol{\omega}
$$

The result is simply obtained applying (38) and (46) recurrently, and can be written symbolically as

$$
\begin{aligned}
& D_{\mathrm{s}_{\kappa_{\alpha} \alpha}^{\alpha}}^{\boldsymbol{\alpha}} \cdots D_{\mathrm{s}_{1}^{\alpha}}^{\boldsymbol{\alpha}} D_{\mathrm{s}_{\kappa_{\omega}}^{\omega}}^{\boldsymbol{\omega}} \cdots D_{\mathrm{s}_{1}^{\omega}}^{\boldsymbol{\omega}} \boldsymbol{\psi}^{E}= \\
& \int_{\boldsymbol{r} \in E(\boldsymbol{\alpha})} \mathcal{D}_{\mathrm{s}_{\kappa_{\alpha}}^{\alpha}}^{E} \cdots \mathcal{D}_{\mathrm{s}_{1}^{\alpha}}^{E} D_{\mathrm{s}_{\kappa_{\omega}}^{\omega}}^{\boldsymbol{\omega}} \cdots D_{\mathrm{s}_{1}^{\omega}}^{\boldsymbol{\omega}} \boldsymbol{\phi}^{E}(\boldsymbol{r}, \boldsymbol{\alpha}, \boldsymbol{\omega}) d E,
\end{aligned}
$$

which should be actually expressed for integration in reference coordinates as

$$
\begin{aligned}
& D_{\mathrm{s}_{\kappa_{\alpha}}^{\alpha}}^{\boldsymbol{\alpha}} \cdots D_{\mathrm{s}_{1}^{\alpha}}^{\boldsymbol{\alpha}} D_{\mathrm{s}_{\kappa_{\omega}}^{\omega}}^{\boldsymbol{\omega}} \cdots D_{\mathrm{s}_{1}^{\omega}}^{\boldsymbol{\omega}} \boldsymbol{\psi}^{E}= \\
& \int_{\boldsymbol{r} \in E(\boldsymbol{\alpha})} \mathcal{D}_{\mathrm{s}_{\kappa_{\alpha}}^{\alpha}}^{\Xi} \cdots \mathcal{D}_{\mathrm{s}_{1}^{\alpha}}^{\Xi} D_{\mathrm{s}_{\kappa_{\omega}}^{\omega}}^{\boldsymbol{\omega}} \cdots D_{\mathrm{s}_{1}^{\omega}}^{\boldsymbol{\omega}} \boldsymbol{\phi}^{\Xi}(\boldsymbol{\xi}, \boldsymbol{\alpha}, \boldsymbol{\omega}) \sqrt{\operatorname{det}[\boldsymbol{G}(\boldsymbol{\xi}, \boldsymbol{\alpha})]} d \Xi .
\end{aligned}
$$

Notice that the shape variation is entirely introduced in the sensitivity analysis by means of the sequential directional derivatives of the jacobian matrix (26) of the transformation, that is, through the sequential directional derivatives of the nodal coordinates $\boldsymbol{r}^{\text {inode }}(\boldsymbol{\alpha})$, that must be known in advance up to the order 


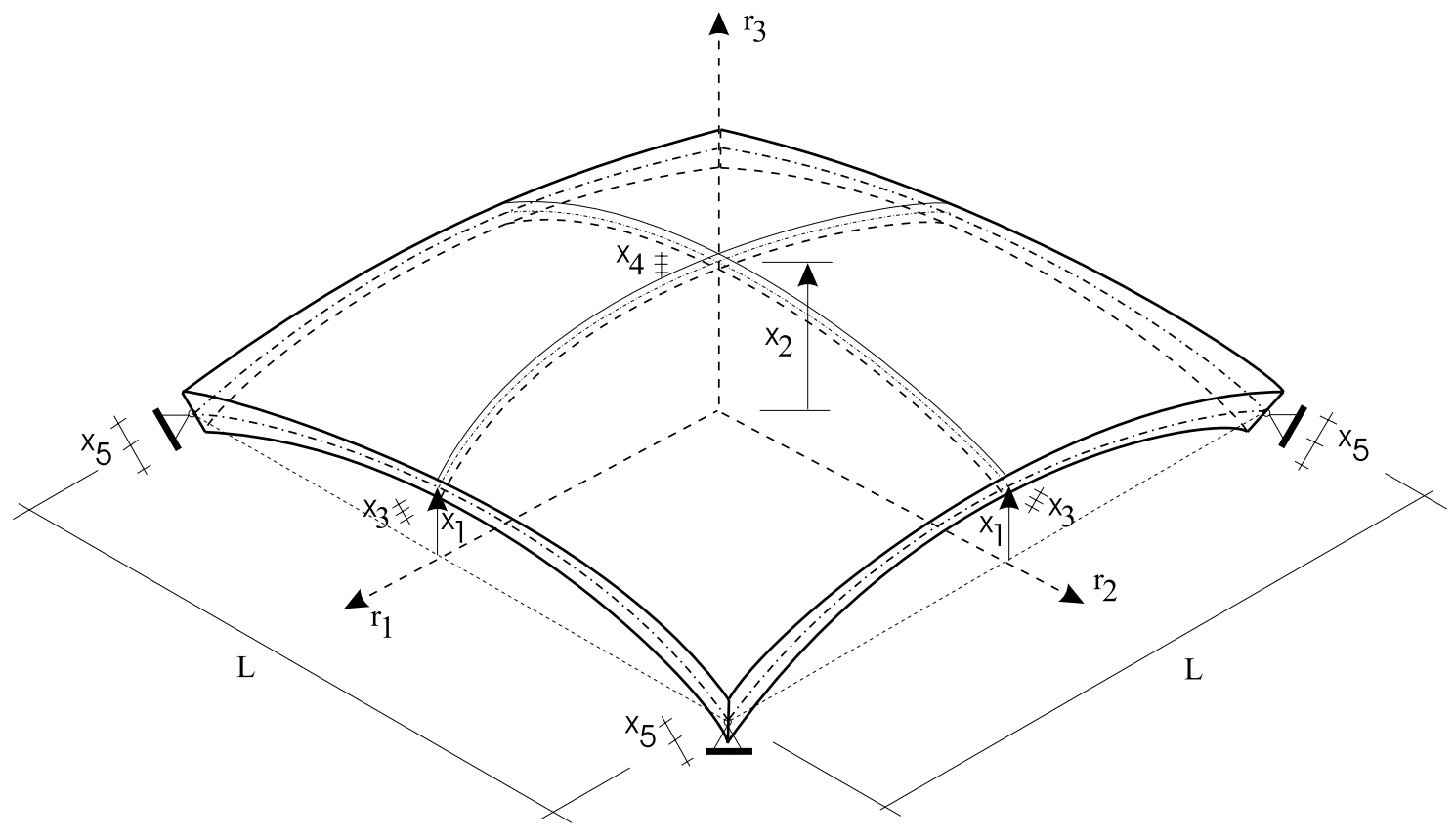

Fig. 2. Design Model

$k$. For $\kappa \leq k$, the $\kappa$-order directional derivative of the jacobian matrix (26) along directions $\boldsymbol{s}_{1}, \ldots, \boldsymbol{s}_{\kappa}$ in the space of design variables can be obviously written as:

$$
D_{\mathrm{s}_{\kappa}^{\alpha}}^{\boldsymbol{\alpha}} \cdots D_{\mathrm{s}_{1}^{\alpha}}^{\boldsymbol{\alpha}} \boldsymbol{J}(\boldsymbol{\xi}, \boldsymbol{\alpha})=\sum_{i \text { node=1 }}^{\text {nnode }} D_{\mathrm{s}_{\kappa}^{\alpha}}^{\boldsymbol{\alpha}} \cdots D_{\mathrm{s}_{1}^{\alpha}}^{\boldsymbol{\alpha}} \boldsymbol{r}^{\text {inode }}(\boldsymbol{\alpha}) \frac{\partial}{\partial \boldsymbol{\xi}} N^{\text {inode }}(\boldsymbol{\xi})
$$

\section{APPLICATION EXAMPLE}

In this example we present the sensitivity analysis performed during the shape optimization of a 3D structure: a concrete roof spanning over a square room and supported on its four vertices (see Figure 2). The shape of the roof is defined by its mid-surface and the thickness distribution. Thus, over a point of material coordinates $\left(r_{1}, r_{2}, 0\right)$, the height of the mid-surface $r_{3}\left(r_{1}, r_{2}\right)$ (measured from the plane of the supports) and the corresponding half-thickness of the wall $\delta\left(r_{1}, r_{2}\right)$ are modelled as

$$
\begin{aligned}
& r_{3}\left(r_{1}, r_{2}\right)=x_{2}+\left(x_{1}-x_{2}\right)\left[\frac{r_{1}^{2}+r_{2}^{2}}{L^{2}}\right]+\left(x_{2}-2 x_{1}\right)\left[\frac{r_{1}^{2} r_{2}^{2}}{L^{4}}\right], \\
& \delta\left(r_{1}, r_{2}\right)=x_{4}+\left(x_{3}-x_{4}\right)\left[\frac{r_{1}^{2}+r_{2}^{2}}{L^{2}}\right]+\left(x_{5}+x_{4}-2 x_{3}\right)\left[\frac{r_{1}^{2} r_{2}^{2}}{L^{4}}\right],
\end{aligned}
$$

for given values of the design variables $\left\{x_{1}, x_{2}, x_{3}, x_{4}, x_{5}\right\}$. The outside and inside surfaces of the roof are generated by carrying the half-thickness of the 

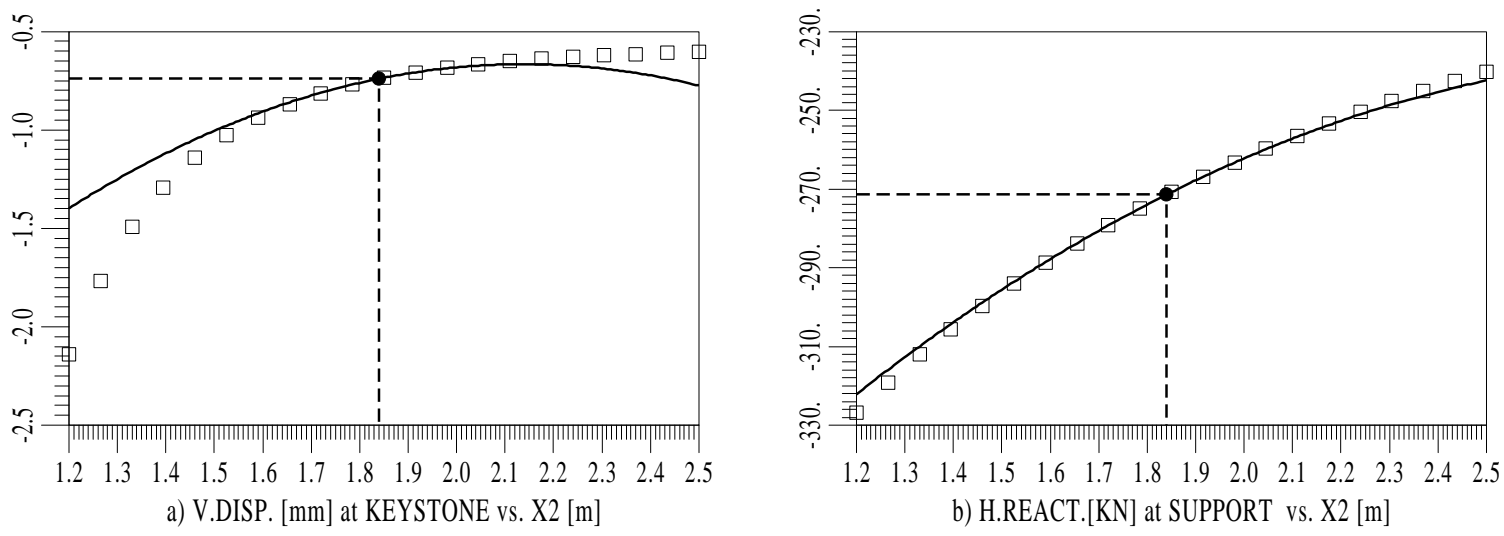

Fig. 3. FEM second order predicted values (solid lines) and FEM computed results (squares) of the following control variables: vertical displacement at the keystone (a), and horizontal reaction at the support (b), versus design variable $x_{2}$. [Load case $1 ; x_{2}^{\text {opt }}=1.83992 \mathrm{~m}$ (circles)]

wall over the mid-surface normal vector. As geometric side constraints we impose

$$
x_{1} \geq 0.000 \mathrm{~m}, x_{2} \geq 0.000 \mathrm{~m}, x_{3} \geq 0.050 \mathrm{~m}, x_{4} \geq 0.050 \mathrm{~m}, x_{5} \geq 0.075 \mathrm{~m},(55)
$$

in order to avoid geometrically unfeasible designs, ensure the roof mid-surface to be entirely over the supports plane, and limit the minimum thickness of the wall. As design constants we choose $L=12 \mathrm{~m}(\operatorname{span}), q_{s}=0.784 \mathrm{KPa}$ (snow load), $E=0.294 \cdot 10^{8} \mathrm{KPa}$ (Young modulus), $\nu=0$ (Poisson modulus), and $\rho_{c}=0.23 \cdot 10^{4} \mathrm{Kg} / \mathrm{m}^{3}$ (density of concrete). The objective function is the weight of the roof. As load cases we consider self weight (case 1), and self weight plus snow load (case 2). We state that

$$
\sigma_{I} \leq 0.000 \mathrm{KPa}, \quad \sigma_{I I I} \geq-980.000 \mathrm{KPa}
$$

to limit the maximum allowable tension $\left(\sigma_{I}\right)$ and compression $\left(\sigma_{I I I}\right)$ in both load cases.

The structural behaviour is analyzed by a linear elastic three-dimensional FEM model. Because of symmetry, only a quarter of the roof is discretized in 20-nodes 3D isoparametric elements. Null displacements are prescribed at the supports. Integration is performed by Gauss quadratures, using $3 \times 3 \times 3$ points for the $3 \mathrm{D}$ elements and $3 \times 3$ points for their boundaries. The stress constraints are imposed at the Gauss integration points located at the center of the upper and lower layers of each element. The results presented in this paper were obtained with a mesh of $3 \times 3 \times 1$ elements. Therefore, 72 non-linear inequality constraints were imposed (considering both load cases). A tolerance of $0.490 \mathrm{KPa}$ was accepted in the stress constraints violation. 

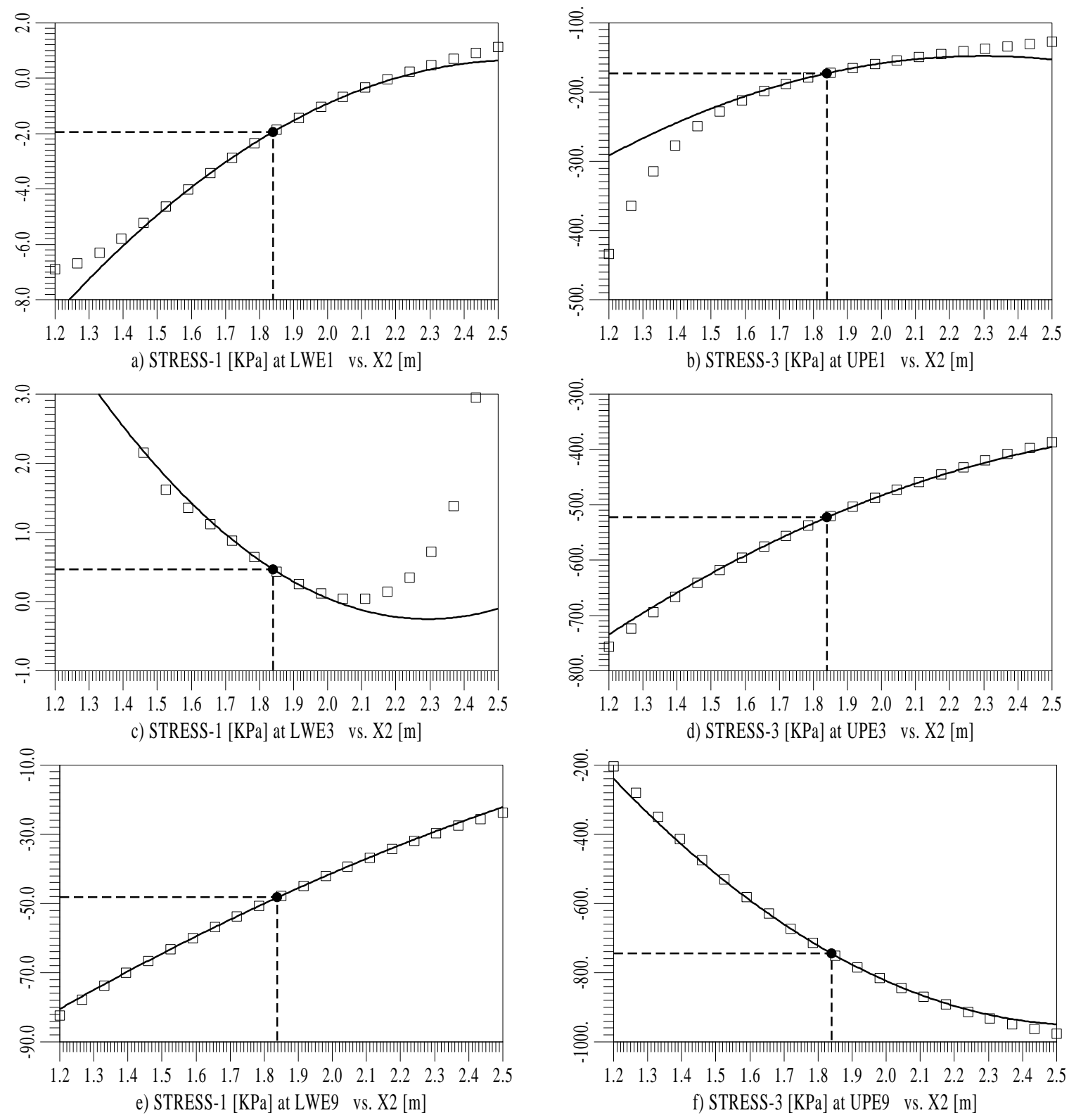

Fig. 4. FEM second order predicted values (solid lines) and FEM computed results (squares) of the following control variables: $\sigma_{I}$ at Gauss points LWE1 (a), LWE3 (c) and LWE9 (e), and $\sigma_{I I I}$ at Gauss points UPE1 (b), UPE3 (d) and UPE9 (f), versus design variable $x_{2}$. [Load case $1 ; x_{2}^{\text {opt }}=1.83992 \mathrm{~m}$ (circles)]

In Figures 3 and 4 we compare some predicted values (obtained from the second order sensitivity analysis at the optimal solution) with the corresponding FEM computed results for different values of the design variable $x_{2}$ around the optimum.

Namely, we compare the corresponding values of the vertical displacement at the keystone (see Figure 3a), the horizontal reaction at the supports (see Figure $3 \mathrm{~b}$ ), and the 1 st and $3 \mathrm{rd}$ principal stresses at several points in which constraints are imposed (see Figure 4) for load case number 1. (Note: LWE\# 
and UPE\# respectively stand for the Gauss points located at the center of the lower and upper layers of element number \#; being element number 1 the closest to the keystone, element number 9 the closest to the support, and element number 3 the closest to the center of one of the free borders.)

The optimization process was performed by the $D A O^{2}$ computer aided optimum design system [9] developed by the authors, giving the optimal solution

$$
x_{1}=0.680 \mathrm{~m}, x_{2}=1.840 \mathrm{~m}, x_{3}=0.050 \mathrm{~m}, x_{4}=0.050 \mathrm{~m}, x_{5}=0.075 \mathrm{~m} .(57)
$$

As shown in Figures 3 and 4, the quality of the quadratic approximations of the structural behaviour (obtained by high order sensitivity analysis) explains the efficiency, reliability and robustness of the $D A O^{2}$ system [6,9]. A detailed description of this optimization problem can be found in [6]. A description of the proposed MP algorithm can be found in [10,11].

\section{APPENDIX I.- A General Expression of the Hypersurface Ele- ment for Integration in Manifolds}

Let $E$ be an open domain in $\mathbb{R}^{n_{r}}$ of dimension $n_{\xi} \leq n_{r}$, and let $\Xi$ be an open reference domain in $\mathbb{R}^{n_{\xi}}$, such that $\bar{E}$ is the image of $\Xi$ by the invertible differentiable mapping

$$
\begin{array}{cc}
\boldsymbol{\rho}: \bar{\Xi} \subset \mathbb{R}^{n_{\xi}} \longrightarrow \bar{E} \subset \mathbb{R}^{n_{r}} \\
\boldsymbol{\xi} & \boldsymbol{r}=\boldsymbol{\rho}(\boldsymbol{\xi})
\end{array}
$$

Let $\boldsymbol{\xi}$ be an arbitrary point in $\bar{\Xi}$ given by its reference coordinates

$$
\boldsymbol{\xi}=\left\{\xi_{i}\right\}, \quad i=1, \ldots, n_{\xi}=\operatorname{dim}(E),
$$

and let $\boldsymbol{r}=\boldsymbol{\rho}(\boldsymbol{\xi})$ be the corresponding point in $\bar{E}$ given by its material coordinates

$$
\boldsymbol{r}=\left\{r_{i}\right\}, \quad i=1, \ldots, n_{r}
$$

Let $\mathrm{d} \boldsymbol{\xi}$ be an arbitrary infinitesimal vector in $\mathbb{R}^{n_{\boldsymbol{\xi}}}$, and let $\mathrm{d} \boldsymbol{r}$ be the corresponding infinitesimal vector in $\mathbb{R}^{n_{r}}$ that joins the point $\boldsymbol{r}=\boldsymbol{\rho}(\boldsymbol{\xi})$ to the point $\boldsymbol{r}+\mathrm{d} \boldsymbol{r}=\boldsymbol{\rho}(\boldsymbol{\xi}+\mathrm{d} \boldsymbol{\xi})$ (see Figure 5$)$. Therefore we can write

$$
\mathrm{d} \boldsymbol{r}=\boldsymbol{J}(\boldsymbol{\xi}) \mathrm{d} \boldsymbol{\xi}, \quad \boldsymbol{J}(\boldsymbol{\xi})=\frac{\partial \boldsymbol{\rho}(\boldsymbol{\xi})}{\partial \boldsymbol{\xi}},
$$


where $\boldsymbol{J}(\boldsymbol{\xi})$ is the jacobian matrix of the mapping. Then, the distance $d s$ between the point $\boldsymbol{r}=\boldsymbol{\rho}(\boldsymbol{\xi})$ and the point $\boldsymbol{r}+\mathrm{d} \boldsymbol{r}=\boldsymbol{\rho}(\boldsymbol{\xi}+\mathrm{d} \boldsymbol{\xi})$ is given by the modulus of the infinitesimal vector $\mathrm{d} \boldsymbol{r}$. Thus

$$
d s=\sqrt{\mathrm{d} \boldsymbol{r}^{T} \mathrm{~d} \boldsymbol{r}}=\sqrt{\mathrm{d} \boldsymbol{\xi}^{T} \boldsymbol{G}(\boldsymbol{\xi}) \mathrm{d} \boldsymbol{\xi}}, \quad \boldsymbol{G}(\boldsymbol{\xi})=\left[\boldsymbol{J}^{T}(\boldsymbol{\xi}) \boldsymbol{J}(\boldsymbol{\xi})\right]
$$

where the matrix $\boldsymbol{G}(\boldsymbol{\xi})$ is the so-called metric tensor, which is required to be positive-definite for the mapping (58) to be acceptable. According to the above expression [22] $E$ is said to be a riemannian $n_{\xi}$-dimensional manifold in $\mathbb{R}^{n_{r}}$.

Let the so-called natural vector of the reference coordinate $\boldsymbol{\xi}_{i}$ at $\boldsymbol{r}=\boldsymbol{\rho}(\boldsymbol{\xi})$ be

$$
\boldsymbol{t}_{i}(\boldsymbol{\xi})=\frac{\partial \boldsymbol{\rho}(\boldsymbol{\xi})}{\partial \boldsymbol{\xi}_{i}}, \quad i=1, \ldots, n_{\xi}
$$

Obviously, each natural vector is tangent to its corresponding coordinate curve (see Figure 5). In these terms, the jacobian matrix (61) can be written as

$$
\boldsymbol{J}(\boldsymbol{\xi})=\left[\frac{\partial \boldsymbol{\rho}(\boldsymbol{\xi})}{\partial \boldsymbol{\xi}_{1}} \cdots \frac{\partial \boldsymbol{\rho}(\boldsymbol{\xi})}{\partial \boldsymbol{\xi}_{n_{\xi}}}\right]=\left[\boldsymbol{t}_{1}(\boldsymbol{\xi}) \cdots t_{n_{\xi}}(\boldsymbol{\xi})\right]
$$

and the coefficients of the metric tensor defined in (62) are

$$
\boldsymbol{G}(\boldsymbol{\xi})=\left[g_{i j}(\boldsymbol{\xi})\right], \quad g_{i j}(\boldsymbol{\xi})=\boldsymbol{t}_{i}^{T}(\boldsymbol{\xi}) \boldsymbol{t}_{j}(\boldsymbol{\xi}), \quad\left\{\begin{array}{l}
i=1, \ldots, n_{\xi} \\
j=1, \ldots, n_{\xi}
\end{array}\right.
$$

We are now interested in obtaining an expression for the " $n_{\xi}$-dimensional hypersurface element in $\mathbb{R}^{n_{r}}$ ", that is the generalized volume $\mathrm{d} E$ of the $n_{\xi^{-}}$ dimensional hypercube defined by the infinitesimal vectors $\left\{\boldsymbol{t}_{i}(\boldsymbol{\xi}) \mathrm{d} \xi_{i}\right\}$ for $i=$ $1, \ldots, n_{\xi}$ (see Figure 5).

We shall first show by induction that the generalized volume $\mathrm{d} E_{k}$ of the $k$-dimensional hypercube defined by the infinitesimal vectors $\left\{\boldsymbol{t}_{i}(\boldsymbol{\xi}) \mathrm{d} \xi_{i}\right\}$ for $i=1, \ldots, k \leq n_{\xi}$ is

$$
\mathrm{d} E_{k}=\sqrt{\operatorname{det}\left[\boldsymbol{G}_{k}(\boldsymbol{\xi})\right]} \mathrm{d} \xi_{1} \cdots \mathrm{d} \xi_{k}, \boldsymbol{G}_{k}(\boldsymbol{\xi})=\left[g_{i j}(\boldsymbol{\xi})\right],\left\{\begin{array}{l}
i=1, \ldots, k, \\
j=1, \ldots, k
\end{array}\right.
$$

where

$$
\boldsymbol{G}_{k}(\boldsymbol{\xi})=\left[\boldsymbol{J}_{k}^{T}(\boldsymbol{\xi}) \boldsymbol{J}_{k}(\boldsymbol{\xi})\right], \quad \boldsymbol{J}_{k}(\boldsymbol{\xi})=\left[\boldsymbol{t}_{1}(\boldsymbol{\xi}) \cdots \boldsymbol{t}_{k}(\boldsymbol{\xi})\right]
$$




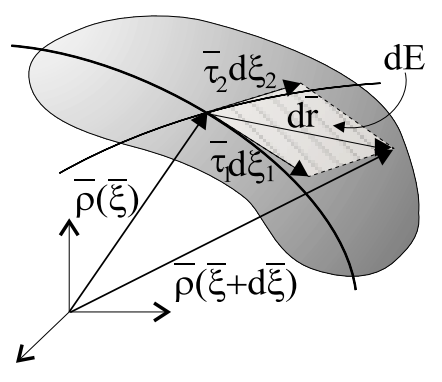

a)

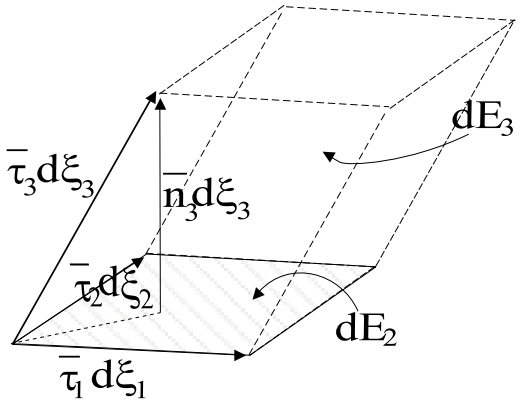

b)

Fig. 5. a) Infinitesimal vectors $\mathrm{d} \boldsymbol{r}$ and $\left\{\boldsymbol{t}_{i} \mathrm{~d} \xi_{i}\right\}$, and 2-dimensional hypersurface element $(\mathrm{d} E)$ in $\mathbb{R}^{3} \cdot \mathrm{b}$ ) Definition of the hypersurface element $\mathrm{d} E_{\kappa+1}$ in terms of the hypersurface element $\mathrm{d} E_{\kappa}$.

It is obvious that (66) holds for the case $k=1$, since the length of the segment (1-dimensional cube) defined by the infinitesimal vector $\left\{\boldsymbol{t}_{1}(\boldsymbol{\xi}) \mathrm{d} \xi_{1}\right\}$ in $\mathbb{R}^{n_{r}}$ is

$$
\mathrm{d} E_{1}=\left|\boldsymbol{t}_{1}(\boldsymbol{\xi})\right| \mathrm{d} \xi_{1}=\sqrt{\boldsymbol{t}_{1}^{T}(\boldsymbol{\xi}) \boldsymbol{t}_{1}(\boldsymbol{\xi})} \mathrm{d} \xi_{1}=\sqrt{\operatorname{det}\left[\boldsymbol{G}_{1}(\boldsymbol{\xi})\right]} \mathrm{d} \xi_{1} .
$$

We shall prove now that if $(66)$ holds for any given $k=\kappa<n_{\xi}$ then $(66)$ holds inmediately for $k=\kappa+1 \leq n_{\xi}$.

Let $\mathrm{d} E_{\kappa}$ be the generalized volume of the $\kappa$-dimensional hypercube defined by the set of infinitesimal vectors $\left\{\boldsymbol{t}_{i}(\boldsymbol{\xi}) \mathrm{d} \xi_{i}\right\}$ for $i=1, \ldots, \kappa<n_{\xi}$. When the infinitesimal vector $\left\{\boldsymbol{t}_{\kappa+1}(\boldsymbol{\xi}) \mathrm{d} \xi_{\kappa+1}\right\}$ is added to the former set, it seems natural to define the generalized volume of the corresponding $(\kappa+1)$-dimensional hypercube as

$$
\mathrm{d} E_{\kappa+1}=\mathrm{d} E_{\kappa}\left|n_{\kappa+1}(\boldsymbol{\xi})\right| \mathrm{d} \xi_{\kappa+1}
$$

where $\boldsymbol{n}_{\kappa+1}(\boldsymbol{\xi})$ is the projection of $\boldsymbol{t}_{\kappa+1}(\boldsymbol{\xi})$ on the orthogonal subspace to the vectors $\boldsymbol{t}_{i}(\boldsymbol{\xi})$ for $i=1, \ldots, \kappa$ (see Figure 5.b). To get an expression for $\left|\boldsymbol{n}_{\kappa+1}(\boldsymbol{\xi})\right|$ we write

$$
\boldsymbol{n}_{\kappa+1}(\boldsymbol{\xi})=\boldsymbol{t}_{\kappa+1}(\boldsymbol{\xi})-\sum_{i=1}^{\kappa} \beta_{i, \kappa+1}(\boldsymbol{\xi}) \boldsymbol{t}_{i}(\boldsymbol{\xi})=\boldsymbol{t}_{\kappa+1}(\boldsymbol{\xi})-\boldsymbol{J}_{\kappa}(\boldsymbol{\xi}) \boldsymbol{\beta}_{\kappa+1}(\boldsymbol{\xi})
$$

where the unknown coefficients $\boldsymbol{\beta}_{\kappa+1}(\boldsymbol{\xi})=\left\{\beta_{i, \kappa+1}(\boldsymbol{\xi})\right\}$ for $i=1, \ldots, \kappa$ must be obtained by imposing that $\boldsymbol{n}_{\kappa+1}(\boldsymbol{\xi})$ be orthogonal to all the vectors $\boldsymbol{t}_{i}(\boldsymbol{\xi})$ for $i=1, \ldots, \kappa$. Thus

$$
\boldsymbol{n}_{\kappa+1}(\boldsymbol{\xi})=\boldsymbol{t}_{\kappa+1}(\boldsymbol{\xi})-\boldsymbol{J}_{\kappa}(\boldsymbol{\xi}) \boldsymbol{G}_{\kappa}^{-1}(\boldsymbol{\xi}) \boldsymbol{g}_{\kappa+1}(\boldsymbol{\xi})
$$


Finally, by means of a Cholesky factorization [25] it can be shown [24] that

$$
\operatorname{det}\left[\boldsymbol{G}_{\kappa}(\boldsymbol{\xi})\right]\left[g_{\kappa+1, \kappa+1}(\boldsymbol{\xi})-\boldsymbol{g}_{\kappa+1}^{T}(\boldsymbol{\xi}) \boldsymbol{G}_{\kappa}^{-1}(\boldsymbol{\xi}) \boldsymbol{g}_{\kappa+1}(\boldsymbol{\xi})\right]=\operatorname{det}\left[\boldsymbol{G}_{\kappa+1}(\boldsymbol{\xi})\right]
$$

which completes the proof, since if (66) holds for $k=\kappa$, introducing (71) in (69) and taking into account (72) we obtain

$$
\mathrm{d} E_{\kappa+1}=\sqrt{\operatorname{det}\left[\boldsymbol{G}_{\kappa+1}(\boldsymbol{\xi})\right]} \mathrm{d} \xi_{1} \cdots \mathrm{d} \xi_{\kappa+1}
$$

Therefore $(66)$ holds for all $k>0$. In particular, for $k=n_{\xi}$ we get

$$
\mathrm{d} E=\sqrt{\operatorname{det}[\boldsymbol{G ( \xi )}]} \mathrm{d} \xi_{1} \cdots \mathrm{d} \xi_{n_{\xi}}
$$

\section{CONCLUSIONS}

An unified approach for high order shape design sensitivity analysis has been presented in this paper. The proposed approach is based on a generic procedure for integration in manifolds. An original, comprehensive and straightforward proof of this procedure is given in Appendix I. Thus, we obtain a single, unified, compact expression to compute high order directional shape sensitivity derivatives, independently of the dimension of the material coordinates system and of the dimension of the elements.

The sensitivity analysis is naturally based upon the existence of a transformation that links the material coordinate system with a fixed reference coordinate system. This is not restrictive, because such a transformation does usually exist in a simple form. Moreover, the implementation of this formulation takes advantage of the fact that such a transformation is inherent to FEM and BEM practical implementations.

Special care has been taken on giving the final results in terms of easy-tocompute expressions, and special emphasis has been made in holding recurrence and simplicity of intermediate operations. The proposed scheme does not depend on any particular form of the state equations, and can be applied to both, direct and adjoint state formulations. Thus, its numerical implementation in standard engineering codes should be considered as a straightforward process. 


\section{Acknowledgement}

This work has been partially supported by Grant Numbers TIC-94-1104 and IN96-0119 of the "Comisión Interministerial de Ciencia y Tecnología" (CICYT) of the Spanish Government, Grant Numbers XUGA-11801B94 and XUGA-IN97-MCM of the "Consellería de Educación e Ordenación Universitaria" of the "Xunta de Galicia", and a research fellowship UAC94 of the "Universidad de La Coruña".

\section{References}

[1] Y. Ding, Shape optimization of structures: A literature survey, Comp. and Struc. 624 (1986) 985-1004.

[2] T. Sussman and K.J. Bathe, The gradient of the finite element variational indicator with respect to nodal point coordinates: An explicit calculation and applications in fracture mechanics and mesh optimization, Int. J. Num. Meth. Engrg. 21 (1985) 763-774.

[3] S. Wang, Y. Sun and R.H. Gallagher, Sensitivity analysis in shape optimization of continuum structures, Comp. and Struc. 520 (1985) 855-867.

[4] J. Cea, Conception optimale ou identification de formes. Calcul rapide de la dérivée directionalle de la fonction coût, Math. Modelling and Numerical Analysis 320 (1986) 371-402.

[5] H. Petryk and Z. Mròz, Time derivatives of integrals and functionals defined on varying volume and surface domains, Arch. Mech. 5-6 38 (1986) 697-724.

[6] F. Navarrina, E. Bendito and M. Casteleiro, High order sensitivity analysis in shape optimization problems, Comp. Meth. in App. Mech. and Eng. 75 (1989) $267-281$.

[7] R.E. Ricketts and O.C. Zienkiewicz, Shape optimization of continuum structures, in: E. Atrek, R.H. Gallagher, K.M. Ragsdell and O.C. Zienkiewicz, eds., New Directions in Optimum Structural Design, (John Wiley \& Sons, Chichester, 1984) 139-166.

[8] F. Navarrina, Una Metodología General para Optimización Estructural en Diseño Asistido por Ordenador, (PhD Thesis, Universidad Politécnica de Cataluña, Barcelona, 1987).

[9] F. Navarrina and M. Casteleiro, A General Methodologycal Analysis for Optimum Design, Int. J. Num. Meth. Engrg. 31 (1991) 85-111.

[10] F. Navarrina and M. Casteleiro, An improved SLP algorithm for structural optimization by the Finite Element Method, in: S.N. Atluri and G.Yagawa, 
eds., Computational Mechanics'88, Theory and Applications, Proceedings of the International Conference on Computational Engineering Science ICES-88, Atlanta 1988, Vol. 2 (Springer-Verlag, Berlin, 1988) 45.ix.1-45.ix.2.

[11] F. Navarrina, M. Casteleiro and R. Tarrech, Algoritmos de Programación Matemática para Optimización de Formas en Ingeniería Estructural, in: M. Doblaré, J.M. Correas, E. Alarcón, L. Gavete and M. Pastor, eds., Métodos Numéricos en Ingeniería, Actas del III Congreso de Métodos Numéricos en Ingeniería, SEMNI-96, Zaragoza 1996, (Sociedad Española de Métodos Numéricos en Ingeniería, Barcelona, 1996) 687-696.

[12] R.J. Fletcher, Practical Methods of Optimization, (John Wiley \& Sons, Chichester, 1981).

[13] O.E. Lev, ed., Structural Otimization. Recent Developments and Applications, (American Society of Civil Engineers, New York, 1981).

[14] J.E. Haug, K.K. Choi and V. Komkov, Design Sensitivity Analysis of Structural Systems, (Academic Press, Orlando, 1986).

[15] T.J.R. Hughes, The Finite Element Method, (Prentice Hall, New Jersey, 1987).

[16] C. Johnson, Numerical Solution of Partial Differential Equations by the Finite Element Method, (Cambridge University Press, Cambridge, 1987).

[17] I. Stakgold, Green's Functions and Boundary Value Problems, (John Wiley \& Sons, New York, 1979).

[18] J.T. Oden and G.F. Carey, Finite Elements: Mathematical Aspects (Volume IV), (Prentice Hall, New Jersey, 1983).

[19] J.T. Oden and G.F. Carey, Finite Elements: A Second Course (Volume II), (Prentice Hall, New Jersey, 1983).

[20] E.B. Becker, G.F. Carey and J.T. Oden, Finite Elements: An Introduction (Volume I), (Prentice Hall, New Jersey, 1981).

[21] M. Spivak, Cálculo en Variedades, Editorial Reverté, Barcelona, 1988).

[22] M.P. Do Carmo, Geometría Diferencial de Curvas y Superficies, (Alianza Universidad, Madrid, 1990).

[23] R. Courant and F. John, Introducción al Cálculo y al Análisis Matemático (Volumen II), (Limusa, México, 1991).

[24] F. Navarrina, S. López, I. Colominas, E. Bendito and M. Casteleiro, High Order Shape Design Sensitivity: A Unified Approach, in: S.R. Idelsohn, E. Oñate and E. Dvorkin, eds., Computational Mechanics: New Trends and Applications, Proceedings of the IV World Conference on Computational Mechanics, Buenos Aires 1998, Centro Internacional de Métodos Numéricos en Ingeniería CIMNE, Barcelona.

[25] J. Stoer and R. Bulirsch, Introduction to Numerical Analysis, (Springer-Verlag, New York, 1983). 